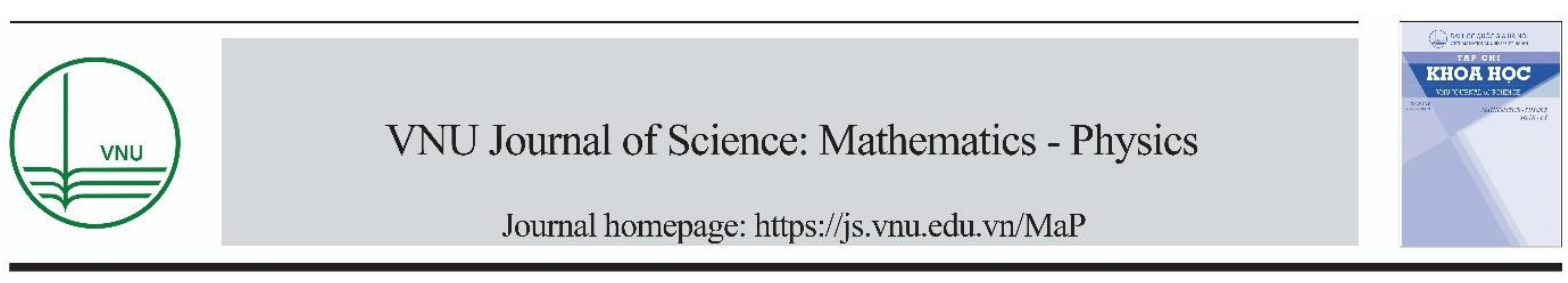

Original Article

\title{
A Note on Invariant Basis Number and Types for Strongly Graded Rings
}

\author{
Nguyen Quang Loc* \\ Hanoi National University of Education, 136 Xuan Thuy, Cau Giay, Hanoi, Vietnam \\ Received 11 April 2020 \\ Revised 03 August 2020; Accepted 15 September 2020
}

\begin{abstract}
Given any pair of positive integers (n, k) and any nontrivial finite group G, we show that there exists a ring $\mathrm{R}$ of type ( $\mathrm{n}, \mathrm{k}$ ) such that $\mathrm{R}$ is strongly graded by $\mathrm{G}$ and the identity component $\mathrm{R}_{\mathrm{e}}$ has Invariant Basis Number. Moreover, for another pair of positive integers $\left(n^{\prime}, k^{\prime}\right)$ with $n \leq n^{\prime}$ and $k \mid k^{\prime}$, it is proved that there exists a ring $R$ of type (n, k) such that $R$ is strongly graded by $G$ and $R_{e}$ has type ( $\left.n^{\prime}, k^{\prime}\right)$. These results were mentioned in [G. Abrams, Invariant basis number and types for strongly graded rings, J. Algebra 237 (2001) 32-37] without proofs.
\end{abstract}

Keywords: Strongly graded ring, Invariant Basis Number, type.

\section{Introduction}

In the study of abstract algebra, most of the rings that one first encounters are rings with Invariant Basis Number property. This class of rings includes all nonzero commutative rings and (left) Noetherian rings. A unital ring $R$ is said to have Invariant Basis Number (IBN) if any two bases of any free left $R$ -module have the same cardinality. Since this condition always holds for free modules with infinite bases (see, e.g., [1, Corollary 1.2]), the definition is equivalent to saying that for any pair of positive integers $m$ and $n, R^{m} \cong R^{n}$ as left $R$-modules only if $m=n$. As a typical example of a ring without IBN, let $V$ be an infinite dimensional vector space over a field $K$ and let $R=\operatorname{End}_{K}(V)$. Then

$$
R=H_{K}(V, V) \cong \operatorname{Hom}_{K}(V \oplus V, V) \cong \operatorname{Hom}_{K}(V, V) \oplus \operatorname{Hom}_{K}(V, V) \cong R \oplus R
$$

\footnotetext{
${ }^{*}$ Corresponding author.

Email address: nqloc@hnue.edu.vn
}

https//doi.org/ 10.25073/2588-1124/vnumap.4507 
as left $R$-modules. As a result, the free left $R$-modules $R^{m}$ and $R^{n}$ are isomorphic for any positive integers $m, n$.

An $R$-homomorphism $R^{n} \rightarrow R^{m}$ can be expressed by an $m \times n$ matrix $A$ with entries in $R$, that is, $A \in \mathrm{M}_{m \times n}(R)$. Thus the ring $R$ fails to have IBN if and only if there exist positive integers $m \neq n$ and matrices $A \in \mathrm{M}_{m \times n}(R), B \in \mathrm{M}_{n \times m}(R)$ such that $A B=I_{m}$ and $B A=I_{n}$. This statement does not involve left nor right modules, so the IBN property is indeed a left-right symmetric condition. It also shows that if there is a unit-preserving ring homomorphism $R^{\prime} \rightarrow R$ and $R$ has IBN, then $R^{\prime}$ has IBN.

If the ring $R$ does not have IBN, then there is a smallest positive integer $n$ for which $R^{n} \cong R^{m}$ as left $R$-modules with some integer $m>n$. We choose $m$ minimal with this property for $n$ and write $m=n+k$ with $k>0$; then we say that $R$ has type $(n, k)$. For example, the ring $R=E n d_{K}(V)$ considered above has type $(1,1)$.

The type of a ring was studied by W. G. Leavitt in the seminal paper [2]. He showed that for any pair of positive integers $(n, k)$, there exists a unital ring of type $(n, k)$ [2, Theorem 8]. Also, the following important results were proved.

Proposition 1.1. ([2, Theorem 2]) Let $R$ and $R^{\prime}$ be unital rings of type $(n, k)$ and $\left(n^{\prime}, k^{\prime}\right)$, respectively. If there is a unit-preserving ring homomorphism $R^{\prime} \rightarrow R$, then $n \leq n^{\prime}$ and $k \mid k^{\prime}$.

Proposition 1.2. ([2, Theorem 3]) Let $R$ and $R^{\prime}$ be unital rings of type $(n, k)$ and $\left(n^{\prime}, k^{\prime}\right)$, respectively. Then $R \oplus R^{\prime}$ has type $\left(\max \left(n, n^{\prime}\right), l c m\left(k, k^{\prime}\right)\right)$. On the other hand, if $R$ and $R^{\prime}$ are IBN rings, then so is $R \oplus R^{\prime}$.

Let $G$ be a multiplicative group with identity element $e$. Recall that a ring $R$ is $G$-graded if $R=\bigoplus_{g \in G} R_{g}$, where each $R_{g}$ is an additive subgroup of $R$ and $R_{g} R_{h} \subseteq R_{g h}$ for all $g, h \in G$. The $G$ graded ring $R$ is called strongly graded if $R_{g} R_{h}=R_{g h}$ for all $g, h \in G$. It is easy to see that the $G$ graded ring $R$ is strongly graded if and only $1_{R} \in R_{g} R_{g^{-1}}$ for any $g \in G$.

For a $G$-graded ring $R$, the identity component $R_{e}$ is a ring and there is a unit-preserving injection $R_{e} \rightarrow R$. Hence if $R$ has IBN, then $R_{e}$ also has IBN by above observation. On the other hand, if the type of $R$ and $R_{e}$ is $\left(n^{\prime}, k^{\prime}\right)$ and $(n, k)$ respectively, then $n^{\prime} \leq n$ and $k^{\prime} \mid k$ by Proposition 1.1.

In [3], the authors asked if $R$ is a $G$-strongly graded ring with $G$ a finite group, whether it is true that $R_{e}$ has IBN if and only if $R$ has IBN. This question was answered in the negative by G. Abrams in $\left[4\right.$, Theorem A], where $G$ is the group $\square_{2}$. Moreover, [4, Theorem B] states that for any pairs of positive integers $(n, k)$ and $\left(n^{\prime}, k^{\prime}\right)$ with $n^{\prime} \leq n$ and $k^{\prime} \mid k$, there exists a ring $R$ of type $\left(n^{\prime}, k^{\prime}\right)$ such that $R$ is strongly graded by $\square_{2}$ and $R_{e}$ has type $(n, k)$. Abrams also mentioned that "it is not hard to show that results analogous to Theorems A and B hold with any finite group $G$ taking the place of $\square_{2}$ " (see [4, page 36]).

The purpose of this paper is to prove Abrams's above remark (see Theorems 3.1 and 3.4). While the construction of the ring $R$ is similar to that of [4], we apply a general method of [5, Theorem 2.10.1] to produce a strong $G$-grading on $R$. To deal with the IBN property and the type, we introduce the 
notion of type of an object in an additive category, which is a generalisation of the corresponding concept for a ring. We show in Proposition 2.2 that how the IBN property or the type of an object $V$ is related to the monogenic monoid genenerated by the isoclass $[V]$. This enables us to obtain a number of useful consequences, among others, Corollary 2.3, which generalises [2, Theorem 1].

We need some more notions on graded modules (see [5] for a complete account on graded rings and modules). Let $R$ be a $G$-graded ring and $M$ be a left $R$-module. The module $M$ is called a graded $R$-module if $M=\bigoplus_{g \in G} M_{g}$, where each $M_{g}$ is an additive subgroup of $M$ and $R_{h} M_{g} \subseteq M_{h g}$ for all $h, g \in G$. A graded homomorphism between graded $R$-modules $M, N$ is an $R$-homomorphism $f: M \rightarrow N$ such that $f\left(M_{g}\right) \subseteq N_{g}$ for all $g \in G$. For a graded $R$-module $M$, $E N D_{R}(M)=\bigoplus_{h \in G} E N D_{R}(M)_{h}$ is a $G$-graded ring, where

$$
\operatorname{END}_{R}(M)_{h}=\left\{f \in \operatorname{End}_{R}(M) \mid f\left(M_{g}\right) \subseteq M_{g h} \text { for all } g \in G\right\} .
$$

The $g$-shifted graded $R$-module $M(g)$ is defined to be $M(g)=\bigoplus_{h \in G} M(g)_{h}$, where $M(g)_{h}=M_{g h}$. We denote by $R$-Mod the category of left $R$-modules and by $R$-Gr the category of graded left $R$-modules with graded homomorphisms.

Throughout this paper, we consider only rings with identity.

\section{The IBN and Types of Objects}

Let us start by extending [3, Definition 3.1] to non-IBN objects.

Definition 2.1. Let $\mathrm{A}$ be an additive category. An object $V \in \mathrm{A}$ has IBN if for all positive integers $m$ and $n, V^{n} \cong V^{m}$ implies $n=m$. If a nonzero object $V \in \mathrm{A}$ does not have $\operatorname{IBN}$, then $V$ has type $(n, k)$ if $n$ is the smallest positive integer such that $V^{n} \cong V^{m}$ for some $m>n$, and $k$ is the smallest positive integer such that $V^{n} \cong V^{n+k}$.

Thus, when $\mathrm{A}=R$-Mod and $V=R$, considered as a left $R$-module, we obtain the usual definitions of IBN property and type for a ring.

Next we point out the relationship between Definition 2.1 and the structure of monogenic semigroups (see [6, Section 1.2]). A monogenic semigroup (or a cyclic semigroup) is a semigroup generated by a single element. Let $S$ be an additive semigroup and $a \in S$. By definition, the monogenic subsemigroup of $S$ generated by $a$ is

$$
\langle a\rangle=\{a, 2 a, 3 a, \ldots\},
$$

where $m a=a+a+\cdots+a$ ( $m$ summands). There are two possibilities for the structure of $\langle a\rangle$. If, whenever $m a=n a$ implies $m=n$ for positive integers $m$ and $n$, then clearly $\langle a\rangle$ is isomorphic to the additive semigroup $\square^{*}$ of positive integers. On the other hand, if $m a=n a$ for some $m \neq n$, then the index of $\langle a\rangle$ is the least positive integer $n$ such that $n a=m a$ for some $m>n$, and the period of $\langle a\rangle$ is the least positive integer $k$ such that $n a=(n+k) a$. In this case, it is proved that

$$
\langle a\rangle=\{a, 2 a, \ldots, n a,(n+1) a, \ldots,(n+k-1) a\}
$$


and $m a=m^{\prime} a$ for $m \neq m^{\prime}$ if and only if $m, m^{\prime} \geq n$ and $m \equiv m^{\prime} \quad(\bmod k)$. The key point here is the elements $n a,(n+1) a, \ldots,(n+k-1) a$ form a group; in particular, these elements are distinct by cancellation law. A monogenic semigroup with index $n$ and period $k$ may also be expressed in terms of semigroup presentation $\{a \mid n a=(n+k) a\}$, which is a free semigroup generated by $a$ subject to the relation $n a=(n+k) a$ (see [6, Section 1.6]).

The above definitions can be carried over monoids (i.e., semigroups with identity) as well, so we may talk about monogenic monoids (where $\langle a\rangle=\{0 a, a, 2 a, 3 a, \ldots\}$ with $0 a$ equal the identity element).

The following proposition is now clear from Definition 2.1 and the previous discussion.

Proposition 2.2. Let $\mathrm{A}$ be an additive category and let $\mathrm{P}$ be a set of objects in $\mathrm{A}$ such that $\mathrm{P}$ is closed under finite direct sums (including the empty sum, which gives the zero object). Let $S$ be the abelian monoid of isomorphism classes of objects in $\mathrm{P}$, where the class of $A \in \mathrm{P}$ is denoted by $[A]$ and the operation on $S$ is defined by

$$
[A]+[B]=[A \oplus B] .
$$

Then, for any object $V \in \mathrm{P}$, we have:

(i) $V$ has IBN if and only if the monogenic submonoid $\langle[V]\rangle$ of $S$ is isomorphic to the additive monoid $\square$

(ii) $V$ has type $(n, k)$ if and only if the monogenic submonoid $\langle[V]\rangle$ of $S$ has index $n$ and period $k$.

As a direct consequence of Proposition 2.2, we obtain the corollary below. This result generalises [2, Theorem 1], which was proved by arguments on bases of free modules and such arguments cannot apply to our more general definition.

Corollary 2.3. Let $\mathrm{A}$ and $\mathrm{P}$ be as in Proposition 2.2. For a pair of positive integers $(n, k)$, an equivalent condition for an object $V \in \mathrm{P}$ to have type $(n, k)$ is:

$$
V^{m} \cong V^{m^{\prime}} \text { for } m \neq m^{\prime} \text { if and only if } m, m^{\prime} \geq n \text { and } m \equiv m^{\prime} \quad(\bmod k) .
$$

Proof. Assume that we have the given condition. Then clearly $V^{n} \cong V^{n+k}$ and $n$ is the smallest positive integer such that $V^{n} \cong V^{m}$ for some $m>n$. If $V^{n} \cong V^{n+h}$ for some $0<h<k$, then we would have $n \equiv n+h \quad(\bmod k)$, which is absurd. Hence the type of $V$ is $(n, k)$. The converse follows from Proposition 2.2.

The next proposition is an extension of [3, Proposition 3.3] to the case of non-IBN objects. By using Corollary 2.3, the proof is similar to that of [3, Proposition 3.3], so we will omit it.

Proposition 2.4. Let $\mathrm{A}$ be an additive category and let $\mathrm{P}$ be a set of objects in $\mathrm{A}$ which is closed under finite direct sums. Then $V \in \mathrm{A}$ has IBN if and only if the ring $\operatorname{End}_{\mathrm{A}}(V)$ has IBN. Similarly, $V \in \mathrm{P}$ has type $(n, k)$ if and only if $\operatorname{End}_{\mathrm{A}}(V)$ has type $(n, k)$.

As an application of the above results, let $R$ be a (unital) ring and take $\mathrm{A}=R$-Mod, $\mathrm{P}$ to be the set of all finitely generated projective left $R$-modules. We denote by $\mathrm{V}(R)$ the monoid of isomorphism classes (denoted by $[P]$ ) of finitely generated projective left $R$-modules under operation given by direct sum. The monoid $\mathrm{V}(R)$ is conical, that is, if $x, y \in \mathrm{V}(R)$ and $x+y=0$, then $x=y=0$. Moreover, $[R]$ is an order-unit in $\mathrm{V}(R)$. Recall that for a monoid $S$, an order-unit in $S$ is an element $u \in S$ such that 
for each $x \in S$, there exist $y \in S$ and an integer $n \geq 1$ such that $x+y=n u$. By a result of Bergman [7, Theorem 6.2], any conical monoid with an order-unit appears as $\mathrm{V}(R)$ for some ring $R$.

From Propositions 2.2 and 2.4 we obtain immediately:

Corollary 2.5. Let $R$ be $a$ ring and $V$ a finitely generated projective $R$-module. Let $M$ denote the submonoid generated by $[V]$ in the monoid $\mathrm{V}(R)$. Then we have:

(i) $V$ has IBN if and only if $\operatorname{End}_{R}(V)$ has IBN, if and only if $M$ is isomorphic to $\square$.

(ii) $V$ has type $(n, k)$ if and only if $\operatorname{End}_{R}(V)$ has type $(n, k)$, if and only if $M$ is a monogenic monoid with index $n$ and period $k$.

Specialising Corollaries 2.5 and 2.3 to the case $V=R$, we recover the following known result.

Corollary 2.6. Let $M$ denote the submonoid generated by $[R]$ in the monoid $\mathrm{V}(R)$. Then we have:

(i) $R$ has IBN if and only if $M$ is isomorphic to $\square$.

(ii) $R$ has type $(n, k)$ if and only if $M$ is a monogenic monoid with index $n$ and period $k$, if and only if

$$
R^{m} \cong R^{m^{\prime}} \text { for } m \neq m^{\prime} \Leftrightarrow m, m^{\prime} \geq n \text { and } m \equiv m^{\prime} \quad(\bmod k)
$$

\section{Strongly Graded Rings of Given Types}

To construct a ring having IBN or having a particular type $(n, k)$, one possible way is using Corollary 2.5. Specifically, we will construct a module $V$ of type $(n, k)$ over some ring $T$ via its monoid realisation. The desired ring will be $\operatorname{End}_{T}(V)$. To show that this ring is strongly graded by a given finite group $G$, we use [5, Theorem 2.10.1]. Let us first recall several related notions in loc. cit.

Let $\mathrm{A}$ be an abelian category and $M, N \in \mathrm{A}$. We say that $N$ weakly divides $M$ in $\mathrm{A}$ if $N$ is isomorphic to a direct summand of a finite direct sum $M^{t}$ of copies of $M$. We say that $M, N$ are weakly isomorphic in A, denoted by $M \sim N$, if and only if they weakly divide each other in A .

In particular, we consider $\mathrm{A}=R$-Gr for a $G$-graded ring $R$. A module $M \in R$-Gr is said to be weakly $G$-invariant if $M \sim M(g)$ in $R$-Gr for all $g \in G$, where $M(g)$ is the $g$-shifted of $M$. Then for a finite group $G,\left[5\right.$, Theorem 2.10.1] states that the $G$-graded $\operatorname{ring} \operatorname{End}_{R}(M)$ is strongly graded if and only if $M$ is weakly $G$-invariant. Note that the finiteness condition imposed on $G$ implies that $\operatorname{End}_{R}(M)=\operatorname{END}_{R}(M)$, thus $\operatorname{End}_{R}(M)$ is a graded ring (see [5, Corollary 2.4.6]).

We are now ready to state and prove the first main theorem of this section.

Theorem 3.1. Let $(n, k)$ be any pair of positive integers and $G$ be any nontrivial finite group. Then there exists a ring $R$ of type $(n, k)$ such that $R$ is strongly graded by $G$ and $R_{e}$ has IBN.

Proof. We follow the idea of [4, Theorem A]. Assume that $|G|=m$. We consider the abelian monoid $(S,+)$ (with identity 0$)$ presented by generators $\left\{x_{1}, x_{2}, \ldots, x_{m}, y_{1}, y_{2}, \ldots, y_{m}\right\}$ and relations

$$
n\left(x_{1}+x_{2}+\cdots+x_{m}\right)=(n+k)\left(x_{1}+x_{2}+\cdots+x_{m}\right) \text {, }
$$




$$
x_{1}+y_{1}=x_{2}, x_{2}+y_{2}=x_{3}, \ldots, x_{m}+y_{m}=x_{1} .
$$

Since the given relations do not have 0 as their right-hand or left-hand sides, the monoid $S$ is conical. Moreover, it is easy to verify that $x_{1}$ is an order-unit of $S$. Therefore, by [7, Theorem 6.2], there exists a ring $T$ such that the monoid $\mathrm{V}(T)$ is isomorphic to $S$. Let $X_{1}, X_{2}, \ldots, X_{m}, Y_{1}, Y_{2}, \ldots, Y_{m}$ denote the finitely generated projective left $T$-modules corresponding to the monoid elements $x_{1}, x_{2}, \ldots, x_{m}, y_{1}, y_{2}, \ldots, y_{m}$, respectively.

We set $V=X_{1} \oplus X_{2} \oplus \cdots \oplus X_{m}$ and $R=\operatorname{End}_{T}(V)$. Then $V$ is a finitely generated projective $T$ module, i.e., $[V] \in \mathrm{V}(T)$. The relation (3.1) shows that the submonoid generated by $[V]$ in $\mathrm{V}(T)$ is isomorphic to a monogenic monoid having presentation $\langle v \mid n v=(n+k) v\rangle$. By Corollary 2.5, both $V$ and $R$ have type $(n, k)$.

Assume that $G=\left\{e, g_{2}, \ldots, g_{m}\right\}$. Let the ring $T$ be graded trivially by $G$, i.e., $T_{e}=T$ and $T_{g}=0$ for all $g \neq e$. The module $V$ is graded as follows:

$$
V=\bigoplus_{g \in G} V_{g} \quad \text { with } \quad V_{e}=X_{1}, V_{g_{2}}=X_{2}, \ldots, V_{g_{m}}=X_{m} .
$$

Clearly $V \in T$-Gr. The cyclic-type relations (3.2) imply that $V_{g}$ and $V_{h}$ are weakly isomorphic in $T$-Mod, for all $g, h \in G$ (precisely, each of them is a direct summand of the other). As a result, $V$ is weakly $G$-invariant. Indeed, for a fixed $g \in G, V_{h}$ is a direct summand of $V(g)_{h}=V_{g h}$ for all $h \in G$; thus $V$ is a direct summand of $V(g)$ in $T$-Gr and vice versa. By [5, Theorem 2.10.1], the ring $R=\operatorname{End}_{T}(V)$ is strongly $G$-graded, as desired.

The identity component $R_{e}$ of $R=\operatorname{End}_{T}(V)=\bigoplus_{g, h \in G} \operatorname{Hom}_{T}\left(V_{g}, V_{h}\right)$ consists of homomorphisms $f$ such that $f\left(V_{g}\right) \subseteq V_{g}$ for all $g \in G$. Hence

$$
R_{e}=\bigoplus_{g \in G} \operatorname{End}_{T}\left(V_{g}\right)=\operatorname{End}_{T}\left(X_{1}\right) \oplus \operatorname{End}_{T}\left(X_{2}\right) \oplus \cdots \oplus \operatorname{End}_{T}\left(X_{m}\right) .
$$

It follows from the construction and Corollary 2.5 that $X_{1}, X_{2}, \ldots, X_{m}$ all have IBN. Consequently, the rings $\operatorname{End}_{T}\left(X_{1}\right), \operatorname{End}_{T}\left(X_{2}\right), \ldots, \operatorname{End}_{T}\left(X_{m}\right)$ have IBN. As a direct sum of IBN rings, the ring $R_{e}$ also has IBN, by Proposition 1.2.

The following lemma is an obvious generalisation of an observation in [4].

Lemma 3.2. Let $\left(n^{\prime}, k^{\prime}\right)$ be any pair of positive integers and $G$ be any finite group. Then there exists a ring $R$ such that it is strongly graded by $G$ and both $R$ and $R_{e}$ have type $\left(n^{\prime}, k^{\prime}\right)$.

Proof. Let $S$ be a unital ring of type $\left(n^{\prime}, k^{\prime}\right)$ and let $R$ be the group ring $S[G]$. Then clearly $R$ is strongly graded by $G$ and $R_{e} \cong S$. In particular, $R_{e}$ has type $\left(n^{\prime}, k^{\prime}\right)$. There are unit-preserving ring homomorphisms from $R_{e}$ to $R$ (the inclusion map) and from $R$ to $R_{e}$ (which maps $\sum_{g \in G} s_{g} g$ to $\left.\left(\sum_{g \in G} s_{g}\right) e\right)$. It follows from Proposition 1.1 that $R$ and $R_{e}$ have the same type $\left(n^{\prime}, k^{\prime}\right)$ 
Proposition 3.3. Let $(n, k)$ be any pair of positive integers and $G$ be any nontrivial finite group. Then there exists a ring $R$ of type $(1,1)$ such that $R$ is strongly graded by $G$ and $R_{e}$ has type $(n, k)$.

Proof. By Lemma 3.2, the proposition holds true in the case $(n, k)=(1,1)$. So we assume that $n k-1>0$. Assume $G=\left\{e, g_{2}, \ldots, g_{m}\right\}$. Let $T$ be a unital ring of type $(n, k)$. We consider the free left $T$-module

$$
V=\underbrace{T \oplus T^{n k-1} \oplus T^{n k} \oplus \cdots \oplus T^{n k}}_{m \text { summands }} \cong T^{(m-1) n k} .
$$

By Corollary 2.6, $V \cong V^{2}$ as left $T$-modules. Thus $V$ has type $(1,1)$. Let $R=\operatorname{End}_{T}(V)$, then $R$ also has type $(1,1)$ by Corollary 2.5 .

Now let the ring $T$ be graded trivially by $G$. The $T$-module $V$ is graded as follows:

$$
V=\bigoplus_{g \in G} V_{g} \quad \text { with } \quad V_{e}=T, V_{g_{2}}=T^{n k-1}, V_{g_{3}}=T^{n k}, \ldots, V_{g_{m}}=T^{n k} .
$$

Clearly $V \in T$-Gr; moreover, $V$ is weakly $G$-invariant. By [5, Theorem 2.10.1], the ring $R=\operatorname{End}_{T}(V)$ is strongly $G$-graded. The identity component $R_{e}$ of $R$ consists of homomorphims $f$ such that $f\left(V_{g}\right) \subseteq V_{g}$ for all $g \in G$. Thus, in the matrix form, we have

$$
R_{e}=\left[\begin{array}{ccccc}
T & 0 & 0 & \cdots & 0 \\
0 & \mathrm{M}_{n k-1}(T) & 0 & \cdots & 0 \\
0 & 0 & \mathrm{M}_{n k}(T) & \cdots & 0 \\
\vdots & \vdots & \vdots & \ddots & \vdots \\
0 & 0 & 0 & \cdots & \mathrm{M}_{n k}(T)
\end{array}\right] .
$$

There are unit-preserving ring homomorphisms from $R_{e}$ to $T$ (the projection from $R_{e}$ into the upper left corner) and from $T$ to $R_{e}$ (the diagonal map). It follows from Proposition 1.1 that $R_{e}$ and $T$ have the same type $(n, k)$.

Using Lemma 3.2 and Proposition 3.3, the proof of the following theorem is similar to that of [4, Theorem B].

Theorem 3.4. Let $\left(n^{\prime}, k^{\prime}\right)$ be any pair of positive integers and $G$ be any nontrivial finite group. For any pair $(n, k)$ of positive integers with $n^{\prime} \leq n$ and $k^{\prime} \mid k$, there exists a ring $R$ of type $\left(n^{\prime}, k^{\prime}\right)$ such that $R$ is strongly graded by $G$ and $R_{e}$ has type $(n, k)$.

Proof. By Proposition 3.3, there exists a ring $T$ which is strongly graded by $G$ in such a way that $T$ has type $(1,1)$ and $T_{e}$ has type $(n, k)$. By Lemma 3.2, for the given pair $\left(n^{\prime}, k^{\prime}\right)$ there exists a $\operatorname{ring} T^{\prime}$ strongly graded by $G$ such that both $T^{\prime}$ and $T_{e}^{\prime}$ have type $\left(n^{\prime}, k^{\prime}\right)$. Let $R=T \oplus T^{\prime}$. Then the ring $R$, with the component-wise grading, is strongly graded by $G$. In particular, we have $R_{e} \cong T_{e} \oplus T_{e}^{\prime}$. Applying Proposition 1.2 yields that the type of $R$ is $\left(\max \left(1, n^{\prime}\right), \operatorname{lcm}\left(1, k^{\prime}\right)\right)=\left(n^{\prime}, k^{\prime}\right)$, while the type of $R_{e}$ is $\left(\max \left(n, n^{\prime}\right), \operatorname{lcm}\left(k, k^{\prime}\right)\right)=(n, k)$.

We conclude this paper with some remarks concerning strongly $G$-graded rings, where $G$ is an arbitrary group (see also [3]). In general, we do not know whether Theorems 3.1 and 3.4 are still true if 
$G$ is any infinite group. However, we are able to prove Theorem 3.1 for $G=\square$ and for any pair $(1, k)$ with $k \geq 1$. Indeed, let $R_{k+1}$ be the directed graph consisting of one vertex and $k+1$ loops. We consider the Leavitt path algebra $L_{K}\left(R_{k+1}\right)$ of $R_{k+1}$ with coefficients in a field $K$ (see [8,9]), which has type $(1, k)$ (in general, it follows from the separative cancellation proved in [9] that the Leavitt path algebra of a finite directed graph either has IBN, or has type $(1, m)$ for some $m \geq 1$; we thank the referee for pointing out this fact). It is well-known that Leavitt path algebras are naturally $\square$-graded. Moreover, since $R_{k+1}$ has no sinks, the algebra $L_{K}\left(R_{k+1}\right)$ is strongly $\square$-graded by [10, Theorem 3.15] (this could also be deduced directly from definition of the $\square$-grading on $L_{K}\left(R_{k+1}\right)$ ). Finally, it follows from the proof of [9, Theorem 5.3] that the identity component $L_{K}\left(R_{k+1}\right)_{0}$ is an ultramatricial $K$-algebra, i.e., it is a direct limit of finite direct products of matrix algebras over $K$. It is known (see, e.g., [1, Exercise 1.17]) that every ultramatricial $K$-algebra has IBN, and so $L_{K}\left(R_{k+1}\right)_{0}$ has IBN. As a conclusion, the algebra $L_{K}\left(R_{k+1}\right)$ is strongly $\square$-graded of type $(1, k)$, whereas its identity component has IBN.

\section{Conclusion}

In this paper, we have considered the IBN property and the type of the identity component $R_{e}$ of a strongly $G$-graded ring $R$ in relationship with the type of $R$, where $G$ is any nontrivial finite group. More concretely, we have shown that for any pair of positive integers $(n, k)$, there exist strongly $G$ graded rings $R$ such that $R$ has type $(n, k)$ and $R_{e}$ has IBN, or $R_{e}$ has a given type $\left(n^{\prime}, k^{\prime}\right)$ with $n \leq n^{\prime}$ and $k \mid k^{\prime}$ (Theorems 3.1 and 3.4).

\section{Acknowledgments}

We would like to express our deep gratitude to the referee for her/his careful reading and valuable suggestions, which led to the final shape of the paper.

\section{References}

[1] T.Y. Lam, Lectures on Modules and Rings, Graduate Texts in Mathematics 189, Springer-Verlag New York, 1999.

[2] W.G. Leavitt, The module type of a ring, Trans. Amer. Math. Soc. 103 (1962) 113-130.

[3] C. Năstăsescu, B. Torrecillas, F. Van Oystaeyen, IBN for graded rings, Comm. Algebra 28 (2000) 1351-1360.

[4] G. Abrams, Invariant basis number and types for strongly graded rings, J. Algebra 237 (2001) 32-37.

[5] C. Năstăsescu, F. Van Oystaeyen, Methods of Graded Rings, Lecture Notes in Mathematics 1836, Springer--Verlag Berlin Heidelberg, 2004.

[6] J.M. Howie, Fundamentals of Semigroup Theory, London Math. Soc. Monographs New Series 12, Oxford University Press, 2003.

[7] G.M. Bergman, Coproducts and some universal ring constructions, Trans. Amer. Math. Soc. 200 (1974) 33-88.

[8] G. Abrams, G. Aranda Pino, The Leavitt path algebra of a graph, J. Algebra 293 (2005) 319-334.

[9] P. Ara, M.A. Moreno, E. Pardo, Nonstable K-theory for graph algebras, Algebr. Represent. Theory 10 (2007) 157 178.

[10] R. Hazrat, The graded structure of Leavitt path algebras, Israel J. Math. 195 (2013) 833-895. 